\title{
Nicotine impact on rat substantia nigra compacta
}

\author{
Sanaa A M Elgayar ${ }^{1}$, Ola A Hussein ${ }^{1}$, Heba A Mubarak ${ }^{1}$, Amany M Ismaiel ${ }^{1}$, Asmaa M.S. Gomaa ${ }^{2}$ \\ ${ }^{1}$ Department of Histology, Faculty of Medicine, Assiut University, Assiut, ${ }^{2}$ Department of Medical Physiology, Faculty of Medicine, Assiut University, \\ Assiut, Egypt
}

\begin{abstract}
Nicotine neuronal interactions exert an adverse potential in some brain regions and a significant link has been established between tobacco smoke/nicotine and vascular impairment. This work addresses nicotine impact on various components of the substantia nigra compacta $(\mathrm{SNc})$ in rat. Twenty adult male Albino rats were divided equally into two groups: Group I, vehicle-control group (received saline [1 $\mathrm{ml} / \mathrm{kg}$ body weight intra peritoneally] for 11 days). Group II; nicotine group (received $1.5 \mathrm{mg} / \mathrm{kg}$ body weight/day Sc) for 11 days. Nicotine levels were detected in the serum. Specimens were taken from the mid brain, processed and examined using biochemical, immunohistochemical, ultrastructural and morphometric techniques. In nicotine group, biochemical analysis revealed reduction in total antioxidant capacity (TAC), decrease in dopamine and malondialdehyde (MDA) levels. The mean number of light cells, and the mean surface area of nerve cells/field were significantly reduced, with an increase of dark cells were found in nicotine group compared to control. Immunoreactivity in nicotine group revealed an increase in neuronal $\alpha$-synuclein, reduction in tyrosine hydroxylase enzyme, an increase in caspase 3 and ultrastructure changes suggestive of neuronal apopto. The blood capillaries were markedly affected. Nicotine induced endothelial and pericytic apoptotic changes, irregular lumena and indistinct endothelial junctional complex. Nicotine administered subcutaneously in a small dose may have a deleterious effect on SNc, mainly involving dopaminergic neurons and blood capillaries. This effect seems to be secondary to an oxidative stress that might be produced by reduced TAC and increased MDA levels.
\end{abstract}

Key words: Nicotine, Substantia nigra, Immunohistochemistry

Received October 9, 2020; 1st Revised December 1, 2020; 2nd Revised December 6, 2020; 3rd Revised December 13, 2020; Accepted December 22, 2020

\section{Introduction}

Nicotine, the psychoactive component of tobacco products, stimulate midbrain dopaminergic neurons by activating nicotinic acetylcholine receptors on the dopaminergic somata and by balancing synaptic sources of information [1].

Nicotine treatment for 7 days, induced many adverse effects in rats. These included deterioration of memory, neu-

\footnotetext{
Corresponding author:

Sanaa A M Elgayar (iD)

Department of Histology, Faculty of Medicine, Assiut University, Assiut 71515, Egypt

E-mail: selgar1@hotmail.com
}

ronal apoptosis, DNA fragmentation, reduced antioxidant enzymes activity and excessive construction of lipid peroxidation and reactive oxygen species [2]. Ceaseless nicotine administration in a high dose $(4 \mathrm{mg} / \mathrm{kg}$ body weight for 4 weeks in subcutaneous injections), might induce hippocampal and striatal neurodegeneration [3]. Long-term nicotine exposure induced damage of cerebellar white matter in rat model system [4]. Besides, a strong relation has been recognized between tobacco smoke/nicotine and vascular impairment. Several studies have shown that nicotine exposure for shortor long duration results in a negative impact on the function and/or structure of the blood-brain barrier (BBB), which consequently would lead to the risk of brain edema and neuro-inflammation. It is reported that nicotine creates a dosedependent change in the BBB permeation of macromolecules 
[5]; alter cerebrovascular flow [6, 7] alters expression or function of BBB-associated proteins $[8,9]$ has deleterious effect on the endothelium; and; increases the permeability of the $\mathrm{BBB}$ via a redistribution of tight junction proteins in cerebral micro-vessels [10]. Hawkins et al. [11], 2004 reported that nicotine-induced BBB damage in a mice model.

In a modern study, by using fluorescence microscopy Huang and his coworkers [12] found that nicotine markedly raised expression of S100B (biomarkers for BBB damage) in the hippocampus following use of $(1.5 \mathrm{mg} / \mathrm{kg} / \mathrm{day}$, of nicotine) (oral delivery) for 3 days (twice per day) [12].

The most prominent function of the pars compacta is motor control; its damage can cause a number of movement disorders including Parkinson's disease and other dyskinesias. Although many previous studies demonstrated the possible effects of nicotine on striatum functions [13, 14], the impact of nicotine on the substantia nigra compacta $(\mathrm{SNc})$ which is one of the major areas producing dopamine, has been marginally addressed. Accordingly, to address these critical issues, we proposed in this novel study to explore nicotine effect on morphology, and biology of the diverse constituents of the SNc.

\section{Materials and Methods}

\section{Chemicals}

Nicotine hydrogen tartrate salt (liquid) was purchased from Sigma-Aldrich (St. Louis, MO, USA) and dissolved in normal saline ( $0.9 \%$ sodium chloride) [15].

\section{Animals}

Twenty adult male Albino rats (weighing 250-300 g) were obtained from Assiut University Animal House. They were housed under standard conditions (light, temperature, food, and water). Animal care and use was in accordance with procedures outlined in the National Institutes of Health Guidelines. All procedures have been approved by the institutional ethics committee of Assiut University (No. 17200506).

Rats were divided equally into two groups as follows:

Group I (Vehicle-control): 10 animals received normal saline injection ( $1 \mathrm{ml} / \mathrm{kg}$ body weight intra peritoneally) for 11 days.

Group II (Nicotine group): 10 animals were injected subcutaneously with nicotine dissolved in normal saline (0.9\% $\mathrm{Na} \mathrm{Cl}$ ) in a dose of $1.5 \mathrm{mg} / \mathrm{kg}$ body weight daily, for 11days [16]. Each rat received a dose of $0.3 \mathrm{mg}$ nicotine in a volume of $0.2 \mathrm{ml}$ solution of saline after adjusting for body weight. Nicotine dose was chosen based on finding that it produces plasma levels of nicotine in rats similar to plasma levels of nicotine found in humans smoking one pack of cigarettes per day. In other word, the dose is close to that from low-tomoderate cigarette smoking [17].

\section{Methods}

\section{Biochemical parameters}

Nicotine levels: At the end of the experiment, levels of nicotine were detected in the serum according to Massadeh et al. [18] by gas chromatography-mass spectrometry (GCMS) extraction method (GC-MS apparatus: 7890A-5975B USA-Column DB $5 \mathrm{~ms}$ ) in Analytical Chemistry Unit-Assiut University. The oven temperature program was initially $100^{\circ} \mathrm{C}$ for 1 minute, then $10^{\circ} \mathrm{C} /$ minute to $260^{\circ} \mathrm{C}$ for 1 minute, with the run time 18 minutes. The oven flow program of 1 $\mathrm{ml} /$ minute for 0 minute. The analysis was performed using the selected ion monitoring acquisition mode. The limit of quantitation of nicotine was $(1 \mathrm{ng} / \mathrm{ml})$.

Dopamine concentration: It was detected by high-performance liquid chromatography with electrochemical detection (HPLC-ECD). After sacrifice of the animals, the brains were quickly removed, the left and right striata were dissected out, and kept in liquid nitrogen. Striatal tissue was weighed and placed in $0.2 \mathrm{ml}$ ice cold $0.01 \mathrm{mM}$ perchloric acid containing $0.01 \%$ EDTA and $60 \mathrm{ng}$ 3, 4-dihydroxybenzylamine as an internal standard. Centrifugation $(15,000 \times \mathrm{g}$, 30 minutes, $4^{\circ} \mathrm{C}$ ) was performed and the supernatant was passed through a $0.2 \mu \mathrm{m}$ filter. Twenty microliters of the supernatant were analyzed in the HPLC column $(4.6 \mathrm{~mm} \times 150 \mathrm{~mm}$ C-18 reverse phase column; MC Medical, Tokyo, Japan) by a dual channel coulochem III electrochemical detector (Model 5300; ESA, Inc., Chelmsford, MA, USA) HPLC. Different concentrations of dopamine (DA) standard samples were set to make a standard curve [19].

Determination of serum total antioxidant capacity and serum lipid peroxide or malondialdehyde: Blood samples were obtained from the retro-orbital venous plexus of animals, allowed to clot, centrifuged at 3,000 g for 15 minutes, and the clear, non-hemolyzed supernatant sera were separated for assessment of total antioxidant capacity (TAC) and malondialdehyde (MDA). TAC and MDA were estimated colorimetrically via commercially available kits (Bio-Diagnostics, Giza, Egypt) following the instructions of the kits. Briefly, 
the hydroxyl radical is formed by the Fenton reaction and via the reaction with the colorless substrate O-dianisidine, it forms the bright yellowish-brown dianisyl radical. On addition of a serum, the antioxidant of the serum depressed the oxidative reactions induced by the hydroxyl radicals and preventing the color change and hence providing an effective determination of TAC [20]. Assessment of MDA based on the reaction of thio-barbituric acid with MDA to produce thio-barbituric acid reactive product. The absorbance of this pink product can be detected at $534 \mathrm{~nm}$ [21].

\section{Histological study}

Electron microscopy: The rats were deeply anesthetized with thiopental (50 mg/kg intraperitoneally) (IP) [22]. Animals were perfused trans cardially through the left ventricle with Glutaraldehyde. The brain was removed and the midbrain was excised. SN of midbrain was dissected out using a dissecting microscope, fixed in glutaraldehyde cacodylate buffer (PH 7.4) for 24 hours and post fixed in 1\% osmium tetroxide for 2 hours and then washed in $0.1 \mathrm{~mol} / \mathrm{l}$ phosphate buffer ( $\mathrm{pH}$ 7.4, 15 minutes), and thereafter dehydrated through graded ethanol solutions. The dehydrated blocks were embedded in ethoxy-line resin.

Semithin sections $(0.5-1 \mu \mathrm{m})$ were cut on the ultra-tome and stained with (1\%) toluidine blue (PH 7.3) for light microscopic examination. Ultrathin sections $(50-80 \mathrm{~nm})$ were cut from the selected areas in semithin sections, placed on copper grids, double stained with uranyl acetate and lead citrate [23]. The grids were examined in Assiut University-Electron Microscope Unit, using a transmission electron microscope JEOL (JEM-100 CXII, Tokyo, Japan) and photographed at 80 $\mathrm{KV}$.

Morphometric study: Three non-overlapped fields in each of five semi-thin sections of each of 3 rats from each group were used for measuring the following:

1 - The number of light cells was counted/field in each section $\times 1,000$

2 - The number of dark cells was counted/field in each section $\times 1,000$

3- The surface area of nerve cells area/field $\mu \mathrm{m}^{2}$.
All morphometric studies were done by using image J which is a Java-based open-source image processing package.

Statistical analysis: The morphometric data of each group were expressed as mean \pm standard deviation and statistically analyzed using IBM SPSS Statistics for Windows, Version 20.0 (IBM Co., Armonk, NY, USA). The independent $t$-test was used for comparing the animal groups. A $P$-values of less than 0.05 were considered significant [24].

Immunofluorescence staining: All animals were perfused transcardially with $50 \mathrm{ml}$ of saline $(0.9 \%)$, followed by 200 $\mathrm{ml}$ ice-cold paraformaldehyde $(4 \%)$ in $0.1 \mathrm{M}$, phosphate buffer. The brain was then removed and maintained in the fixative for 24 hours at $4^{\circ} \mathrm{C}$. After an overnight incubation in PBS containing $30 \%$ sucrose at $4^{\circ} \mathrm{C}$, the brain was frozen and then sectioned. Briefly, serial coronal sections of $30 \mu \mathrm{m}$ thickness were cut using a freezing microtome (Leica CM 1800; Leica Microsystems, Bannockburn, IL, USA) on coated slides. Sections were washed in phosphate-buffered saline (PBS) before application of the primary antibody and also between each immunohistochemistry step [23].

The slices were rinsed with PBS for 5 minutes, permeabilized with PBS-0.1\% Triton for 20 minutes, and blocked with PBS containing 3\% bovine serum albumin (Sigma) and $0.3 \%$ Triton X-100 at room temperature for 90 minutes and labeled with the primary antibody for 3 hours at room temperature. Secondary antibody was used diluted in PBS containing $0.2 \%$ BSA for 90 minutes in room temperature. The sections were cover-slipped with mounting media.

The primary antibodies were Rabbit polyclonal antityrosine hydroxylase (1:1,000; Thermo Fisher Scientific, Rockford, IL, USA). Rabbit polyclonal $\alpha$-Synuclein antibody (1:1,000; Thermo Fisher Scientific) and polyclonal anti-caspase-3 antibody (1:100; Chongqing Biopses, 400020, Chongqing, China) were also used. Caspase 3 is formed of a $32 \mathrm{kDa}$ and zymogen that is cleaved into $17 \mathrm{kD}$ and $12 \mathrm{kDa}$ subunits. The immunogen corresponds to the region of the human caspase 3 p 17 subunit. Caspase 3 plays essesntial roles in programed cell death (apoptosis) where it is responsible for DNA fragmentation and chromatin condensation [25].

The secondary antibodies (Goat anti-Rabbit IgG $[\mathrm{H}+\mathrm{L}]$,

Table 1. Nicotine levels in the serum

\begin{tabular}{|c|c|c|c|c|}
\hline & \multicolumn{2}{|c|}{ Control } & \multicolumn{2}{|c|}{ Nicotine } \\
\hline & Range & Mean \pm SD & Range & Mean \pm SD \\
\hline Nicotine levels (ng/ml) & $1.08-1.50$ & $1.26 \pm 0.18$ & $20.0-20.80$ & $20.39 \pm 0.42$ \\
\hline
\end{tabular}

SD, standard deviation. 
Highly Cross-Adsorbed Secondary Antibody, Alexa Fluor 555 [1:300; Thermo Fisher Scientific], Goat anti-Mouse Ig G $[\mathrm{H}+\mathrm{L}]$, Cross-Adsorbed Secondary Antibody, Alexa Fluor 488 [1:300; Thermo Fisher Scientific]).

Fluorescence images were obtained with Olympus fluorescence microscope equipped with fluorescein and rhodamine filters (BX 512007 Tokyo, Japan).

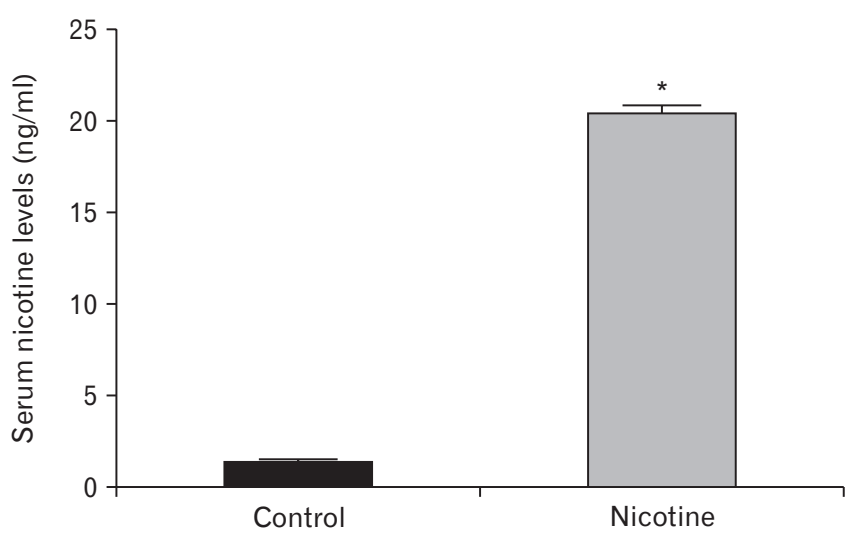

Fig. 1. Serum nicotine levels. Data are expressed as mean \pm standard deviation. * Statistically significant in comparison with the control group $(P<0.05)$.

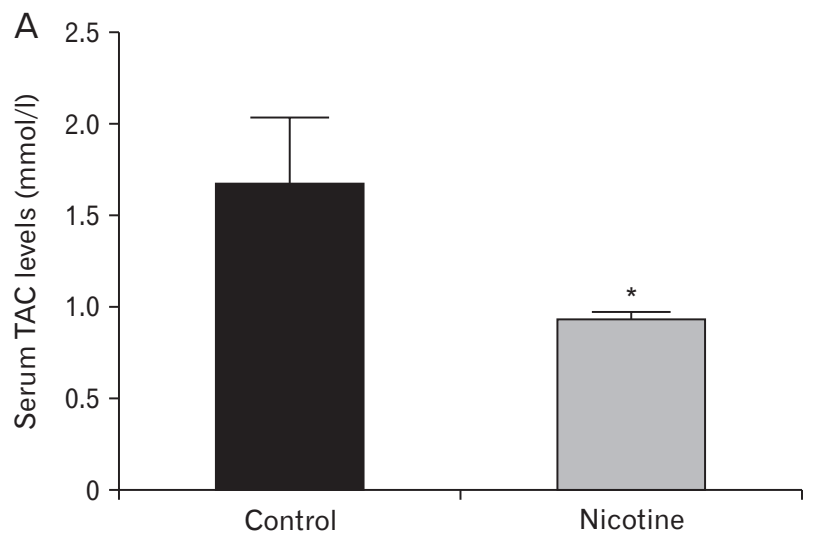

\section{Results}

\section{Bioanlysis}

A significant increased level of nicotine was revealed in the serum of animals in the nicotine group when compared with animals in the control group $(P<0.0001)$ (Table 1, Fig. 1).

A reduction in the striatal dopamine level in plasma was found in nicotine group compared with the control group, however the difference was statistically insignificant $(P>0.05)$ (Table 2). On the other hand, a significant decrease in the level of TAC was observed in the nicotine group when compared with the control group $(P<0.01)$. In addition, a significant increase in the level of MDA was detected in nicotine group compared with the control group $(P<0.01)$ (Fig. 2$)$.

\section{Morphometric results}

Nicotine treated group revealed a significant reduction in the mean number of light cells per field compared to control group ( $P$-value $<0.001)$. In addition, a significant increase of dark cells was detected in nicotine treated group compared to control $(P$-value $<0.001)$ (Fig. 3A). The mean surface area of nerve cells/field was significantly reduced in nicotine treated group compared to control $(P$-value $<0.003)$ (Fig. 3B).

\section{Histological results}

Immunofluorescent staining of control SNc revealed a

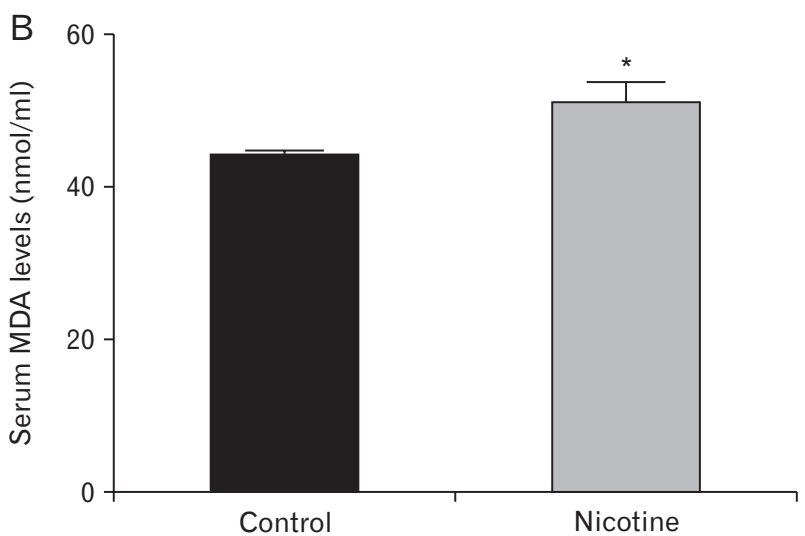

Fig. 2. (A) Serum TAC levels and (B) serum MDA levels. Data are expressed as mean \pm standard deviation. MDA, malondialdehyde; TAC, total antioxidant capacity. ${ }^{*}$ Statistically significant in comparison with the control group $(P<0.05)$.

Table 2. Dopamine levels in the striatum by HPLC (mg/kg tissue)

\begin{tabular}{|c|c|c|c|c|}
\hline & \multicolumn{2}{|c|}{ Control } & \multicolumn{2}{|c|}{ Nicotine } \\
\hline & Range & Mean \pm SD & Range & Mean \pm SD \\
\hline Dopamine levels (ng/mg tissue) & $2.0-2.71$ & $2.44 \pm 0.28$ & $1.99-2.30$ & $2.18 \pm 0.13$ \\
\hline
\end{tabular}

HPLC, high-performance liquid chromatography; SD, standard deviation. 

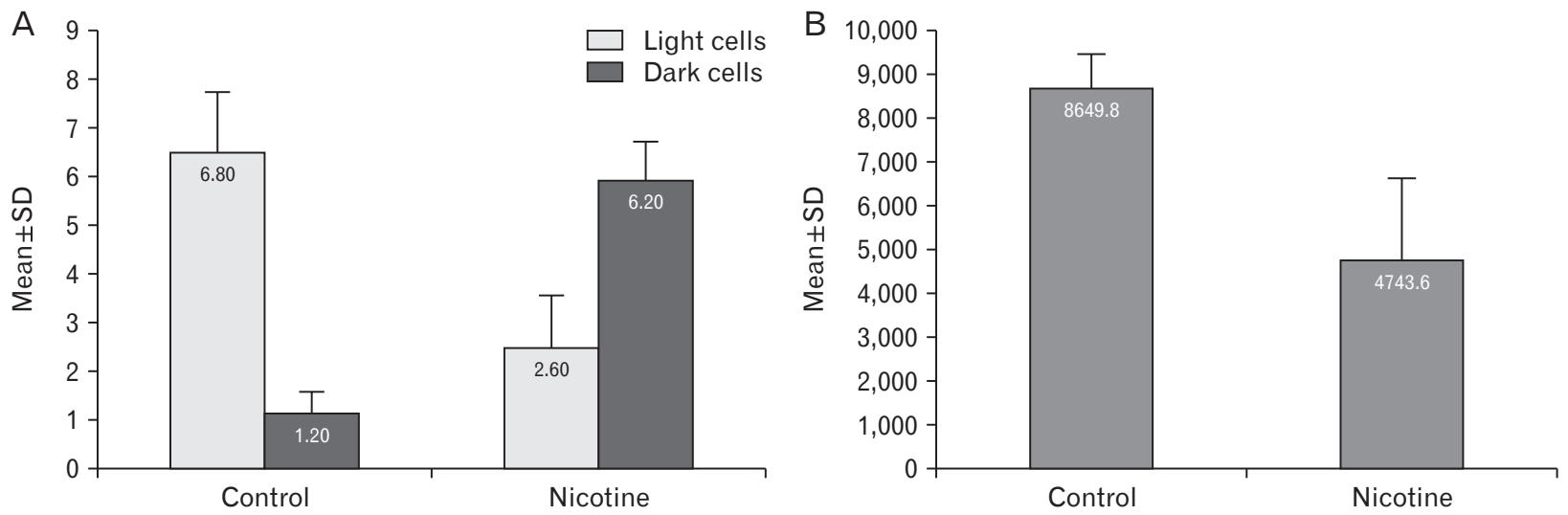

Fig. 3. (A) Number of light and dark cells per field. (B) Surface area of cells/field $\mu \mathrm{m}^{2}$. SD, standard deviation.
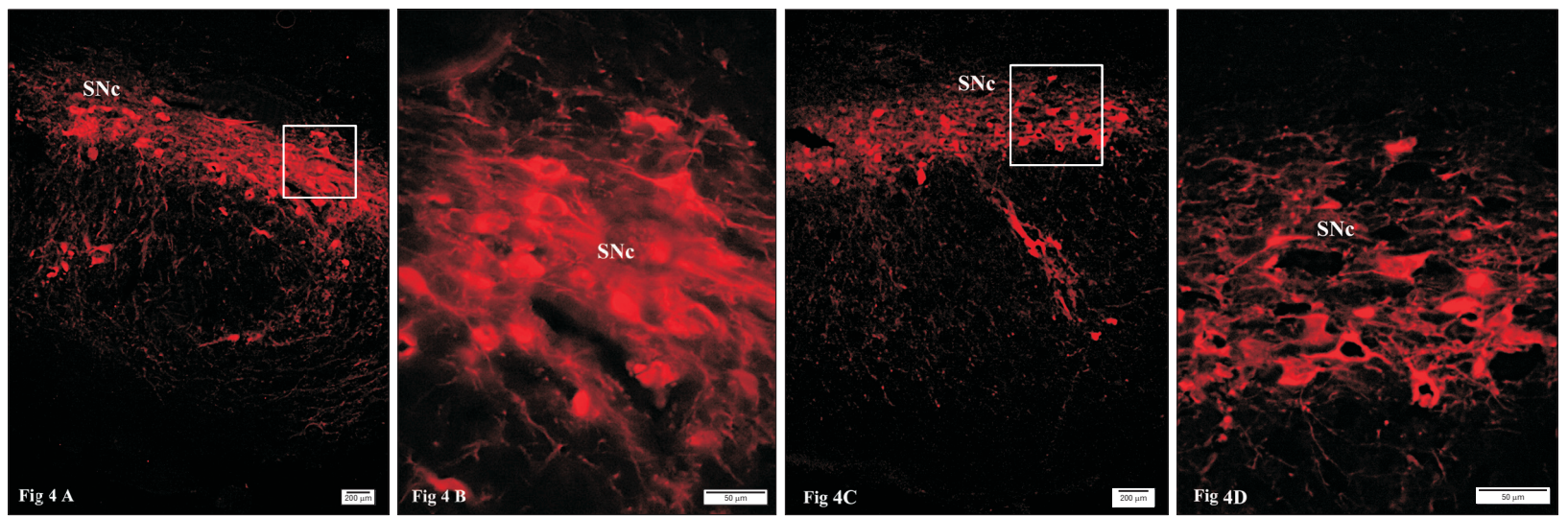

Fig. 4. (A) SNc of control group showing a strong positive immune reaction to TH-antibody. (B) Higher magnification of the previous figure ( $\square$ ). (C) SNc of nicotine group showing a moderate positive immune reaction to TH-antibody. (D) Higher magnification of the previous figure. SNc, substantia nigra compacta; $\mathrm{TH}$, tyrosine hydroxylase.

strong positive reactivity to $\mathrm{TH}$-antibody with numerous TH-positive neurons (Fig. 4A, B). However, this reactivity was less in nicotine-treated group than that detected in control group (Fig. 4C, D). Immunofluorescence staining of caspase 3 was negative in control group (Fig. 5A, B), whereas in nicotine treated group the reaction was positive (Fig. $5 \mathrm{C}, \mathrm{D}$ ). Immunofluorescence for caspase $\alpha$-synuclein antibodies was negative in control group (Fig. 6A, B), whereas in nicotine treated group the reaction was positive (Fig. 6C, D).

In light microscopy, the compacta neurons of control group were closely arranged in the form of a band of flattened cells distal to SNr. They were lightly stained and variable in shapes (spindle, stellate or fusiform), with vesicular nuclei and prominent nucleoli (Fig. 7A). The surrounding neuropil contained numerous blood vessels, glial cells and nerve fibers. In ultrastructure the neurons revealed euchromatic round to oval central nuclei with prominent nucleoli. The surrounding voluminous cytoplasm contained numerous strands of rough endoplasmic reticulum (RER), mitochondria, well developed Golgi bodies and a few small lysosomes (Fig. 7B). The nerve fibers of the control group exhibited regular outlining and contained mitochondria, microtubules, and neurofilaments (Fig. 7A).

In contrary to the control group, the compacta neurons of nicotine treated group were widely separated from each other and most of them were darkly stained with dark nuclei. However, the nucleoli were still distinct within the nuclei and the cytoplasm was metachromatic (Fig. 7A). Ultrastructure revealed many neurons in variable degrees of electrondensity of both the nuclei and cytoplasm with highly prominent nucleoli formed of pars granulosa. Many electron dense neurons revealed a marked increase in cytoplasmic Nissl bodies (Fig. 7B), irregular outlining (blebs) and surrounded by dilated astrocytic processes (Fig. 7C). Other neurons dis- 

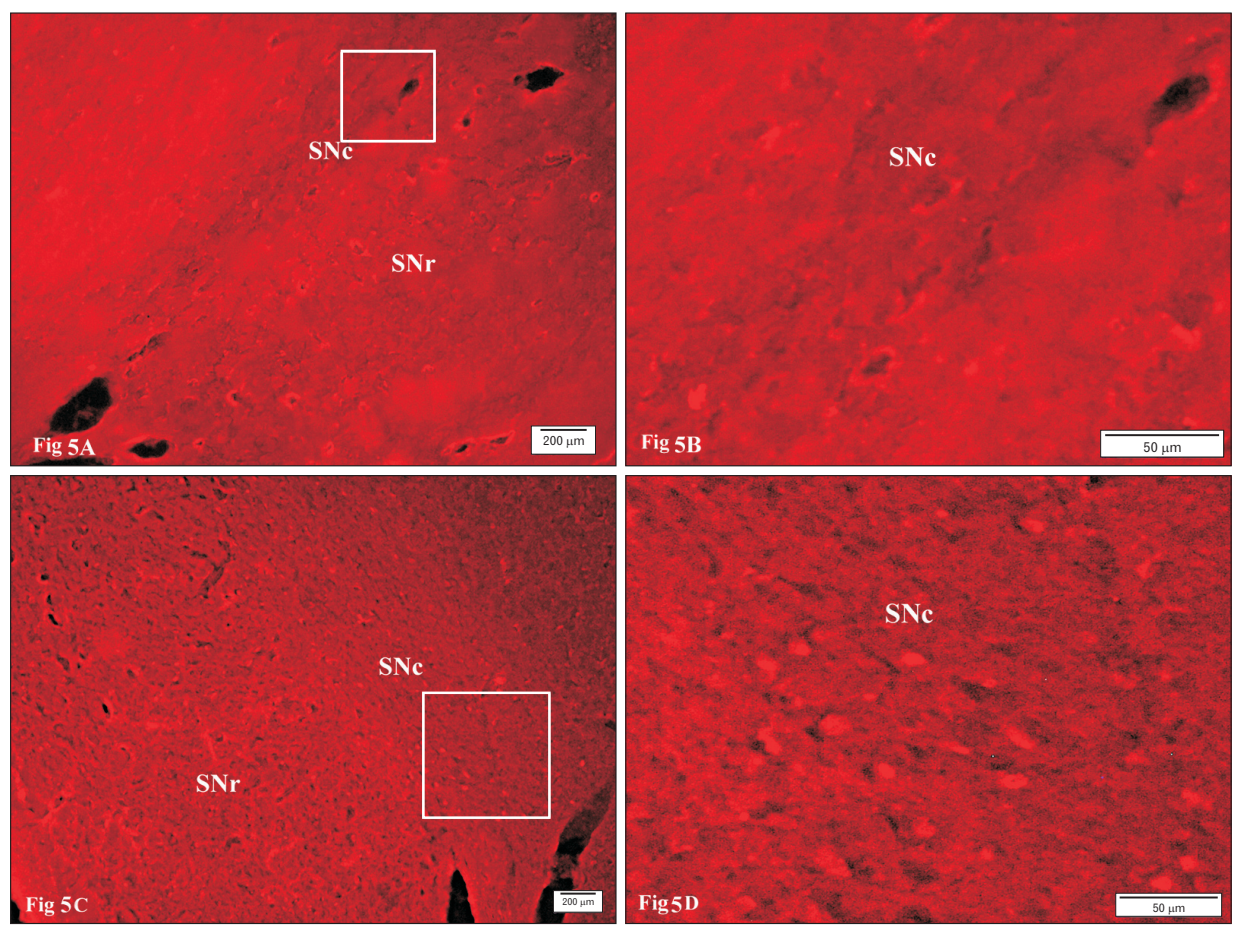

Fig. 5. (A) SNc of control group showing a negative immune reaction to caspase 3 antibody. (B) Higher magnification of the previous figure ( $\square$ ). (C) SNc of nicotine group showing numerous caspase 3 immune positive neurons. (D) Higher magnification of the previous figure ( $\square$ ). SNc, substantia nigra compacta; $\mathrm{SNr}$, substantia nigra reticulata.
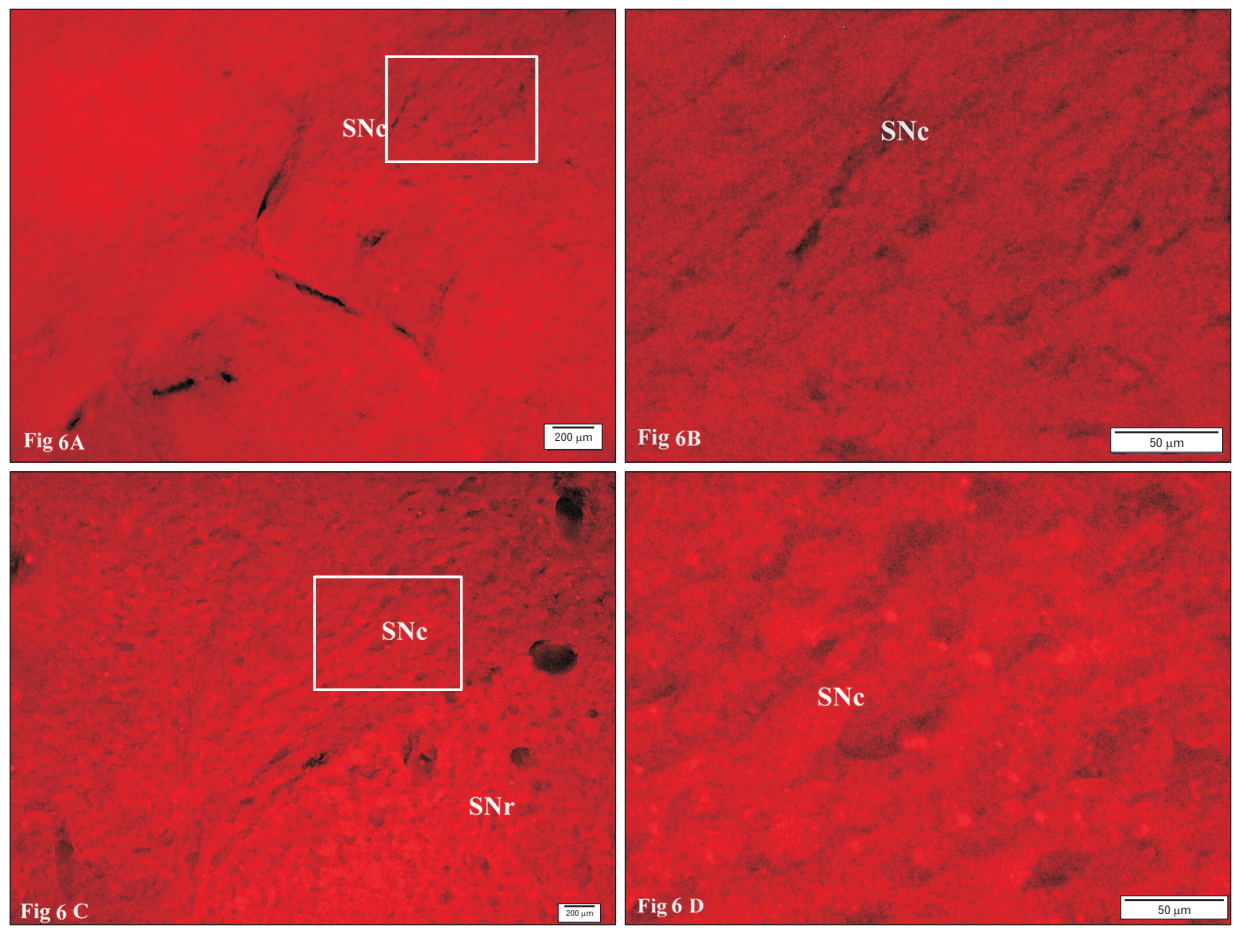

Fig. 6. (A) SNc of control group showing a negative immune reaction to $\alpha$-synuclein antibody. (B) Higher magnification of the previous figure ( $\square$ ). (C) SNc of nicotine group showing a positive immune reaction to $\alpha$-synuclein antibody. (D) Higher magnification of the previous figure $(\square)$. $\mathrm{SNc}$, substantia nigra compacta; $\mathrm{SNr}$, substantia nigra reticularis.

played markedly irregular nuclei, fragmented Golgi and nonmembrane bound frequently-detectable inclusions (Fig. 7D-F). On high magnification most of them contained fibril-like structures (Fig. 7E, F). Occasionally RER cisternae were dilated and contained secretory material (Fig. 7F), which close- ly resembled in shape and electron density, the cytoplasmic inclusions (Fig. 7E, F). Many neuronal processes revealed irregular plasma membrane that formed either corrugated or stepwise surface and variable shaped spine projections (Fig. $7 \mathrm{G}$ ), disorganized microtubules (non-parallel arrangements 

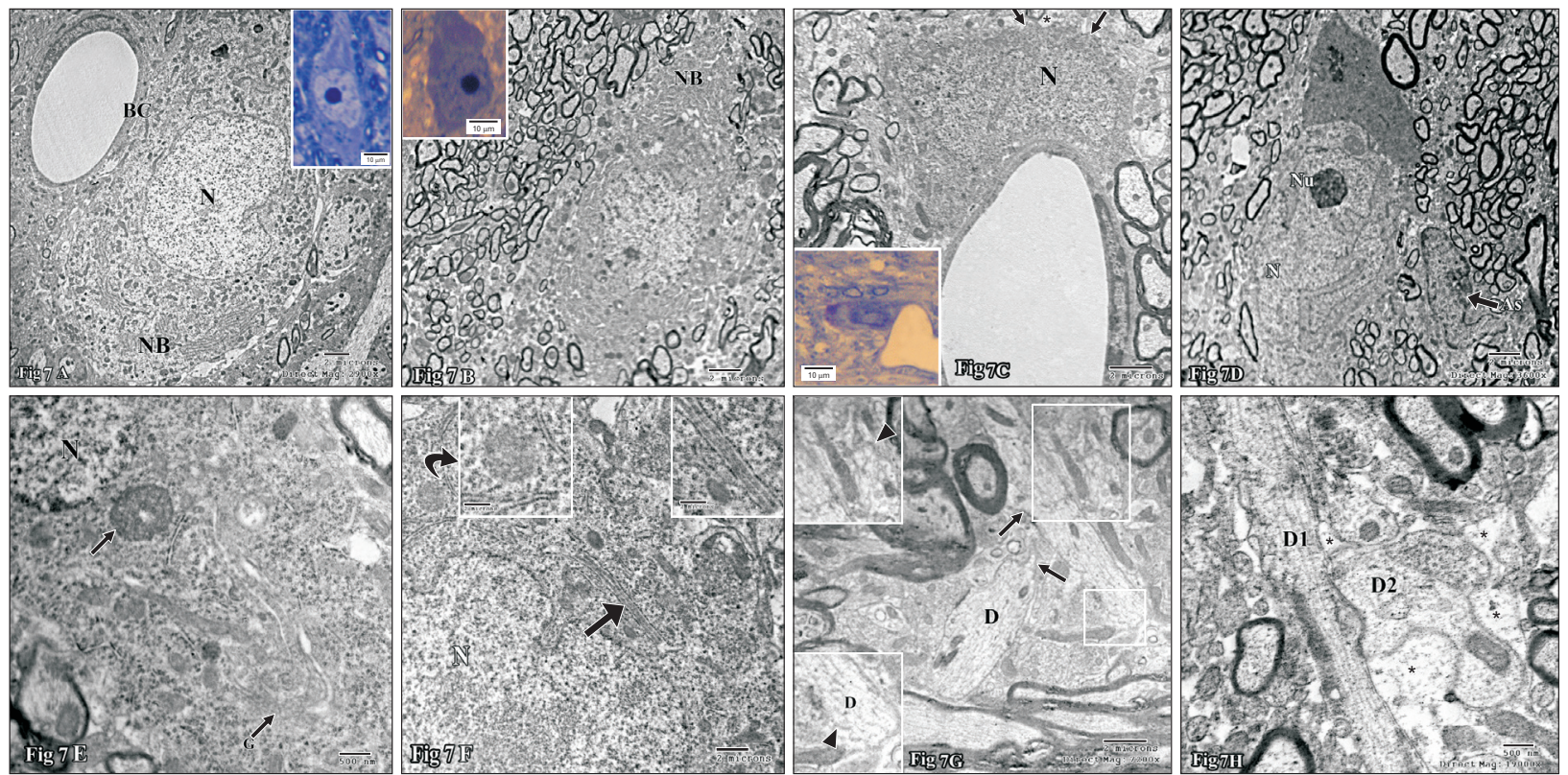

Fig. 7. (A) TEM of SNc of control group showing a neuron with euchromatic N. The cytoplasm contains numerous Nissl bodies and ordinary organelles. Inset: semithin section of SNc of control group sowing a flattened neuron with vesicular $\mathrm{N}$ and well-developed Nu. (B) TEM of $\mathrm{SNc}$ of nicotine group showing a neuron with euchromatic $\mathrm{N}$ and electron dense cytoplasm which contains numerous NBs. Inset: Semithin section of SNc of nicotine group showing a neuron with dark $\mathrm{N}$ and cytoplasm and prominent $\mathrm{Nu}$. (C) TEM of SNc of nicotine group showing a neuron with euchromatic electron dense $\mathrm{N}$, electron dense cytoplasm and irregular outlining (blebbing) ( $\mathbf{\Delta}$ ) surrounded by astrocytic end feet processes. Inset: semithin section for a neuron in a juxta-capillary position. (D) TEM of SNc of nicotine group showing a part of a neuron with euchromatic N. Note the presence of fragmented Golgi body $(\mathrm{G})$ and non-membrane bound inclusion in the cytoplasm ( $\mathbf{A}$ ). (E) TEM of SNc of nicotine group showing a part of a neuron with euchromatic N. Note the presence of secretory material within RER, shown at higher magnification in the right sided inset. Note non-membrane bound inclusion in the cytoplasm shown at a higher magnification in the left sided inset (curved arrow). (F) TEM of SNc of nicotine group showing an As and two adjacent N; one is dense and shrunken, the other possesses an irregular $\mathrm{N}$ and prominent $\mathrm{Nu}$. (G) TEM of SNc of nicotine group showing longitudinally cut dendrites. Note the irregular outer surface ( $\square$ ) of the two adjacent neuronal processes shown at higher magnification in the insets. Spines of variable shapes $(\uparrow)$. (H) TEM of SNc of nicotine group showing a part of a longitudinally cut D1 in synaptic contact with another transversely cut D2. The synaptic terminal is enveloped by astocytic processes $\left(^{*}\right)$. As, astrocyte; BC, blood capillary; D, dendrite; N, nucleus; NBs, Nissl bodies; Nu, nucleolus; RER, rough endoplasmic reticulum; SNc, substantia nigra compacta.
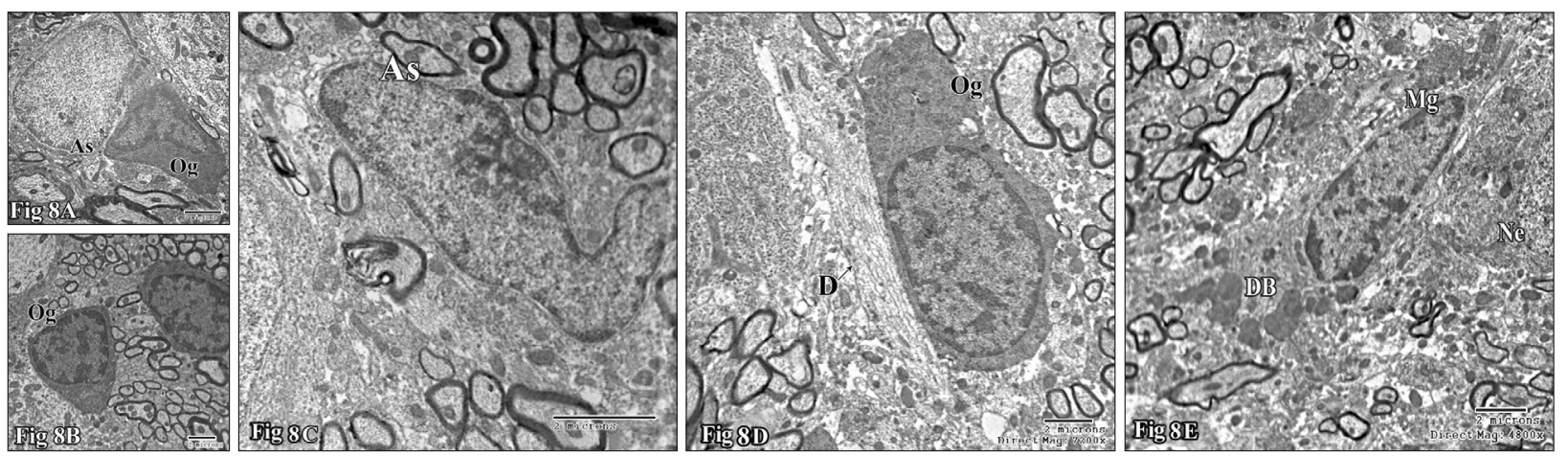

Fig. 8. (A) TEM of SNc of control group showing an As with euchromatic ovoid N, surrounded by a scanty cytoplasm. Note the adjacent Og with moderated electron dense heterochromatic N and cytoplasm. (B) Shows another Og with high electron dense N and cytoplasm. (C) TEM of SNc of nicotine group showing an As with an abnormally large irregular N, prominent Nu surrounded by a thin rim of cytoplasm. (D) TEM of SNc of nicotine group showing an $\mathrm{Og}$ with an elongated $\mathrm{N}$. The adjacent D reveals disorganized microtubules. (E) TEM of SNc of nicotine group showing $\mathrm{Mg}$ with a rod shape $\mathrm{N}$, separated from the adjacent $\mathrm{Ne}$ by astrocytic processes. The cytoplasm contains numerous DB. As, astrocyte; D, dendrite; $\mathrm{DB}$, dense bodies; $\mathrm{Mg}$, microglia; $\mathrm{N}$, nucleus; Og, oligodendrocyte; SNc, substantia nigra compacta. 
or widened intervals) and/ or disrupted microtubules (Fig. $7 \mathrm{H})$. The neuropil revealed numerous dendro-dendritic synapses (Fig. $7 \mathrm{H}$ ).

Neuroglial cells in control group included astrocytes, microglia, and oligodendrocytes. The astrocytes exhibited pale nuclei that were usually regular in shape containing peripheral and some scattered heterochromatin. Their cytoplasm was also pale, scanty and contained a few organelles and extended narrow long processes (Fig. 8A). Microglia cells were found either in relation to blood capillaries (Fig. 8A) or within the tissue parenchyma. They exhibited small nuclei with large heterochromatin, surrounded by a scanty cyto- plasm. Those present contiguous to neurons, their somata were always separated from those of the neurons by astrocytic processes. Regarding oligodendrocyte, two classes may be described within this group of cells; moderately electrondense oligodendrocytes (Fig. 8A), which are large cells with moderately electron dense nucleus and cytoplasm, containing well developed organelles, and giving off a fair number of fine processes; and electron-dense oligodendrocytes, with very dense nucleus and cytoplasm, containing a prominent Golgi bodies (Fig. 8B).

In nicotine-treated group the astrocytes were reactive, hypertrophied and abnormally shaped nuclei surrounded
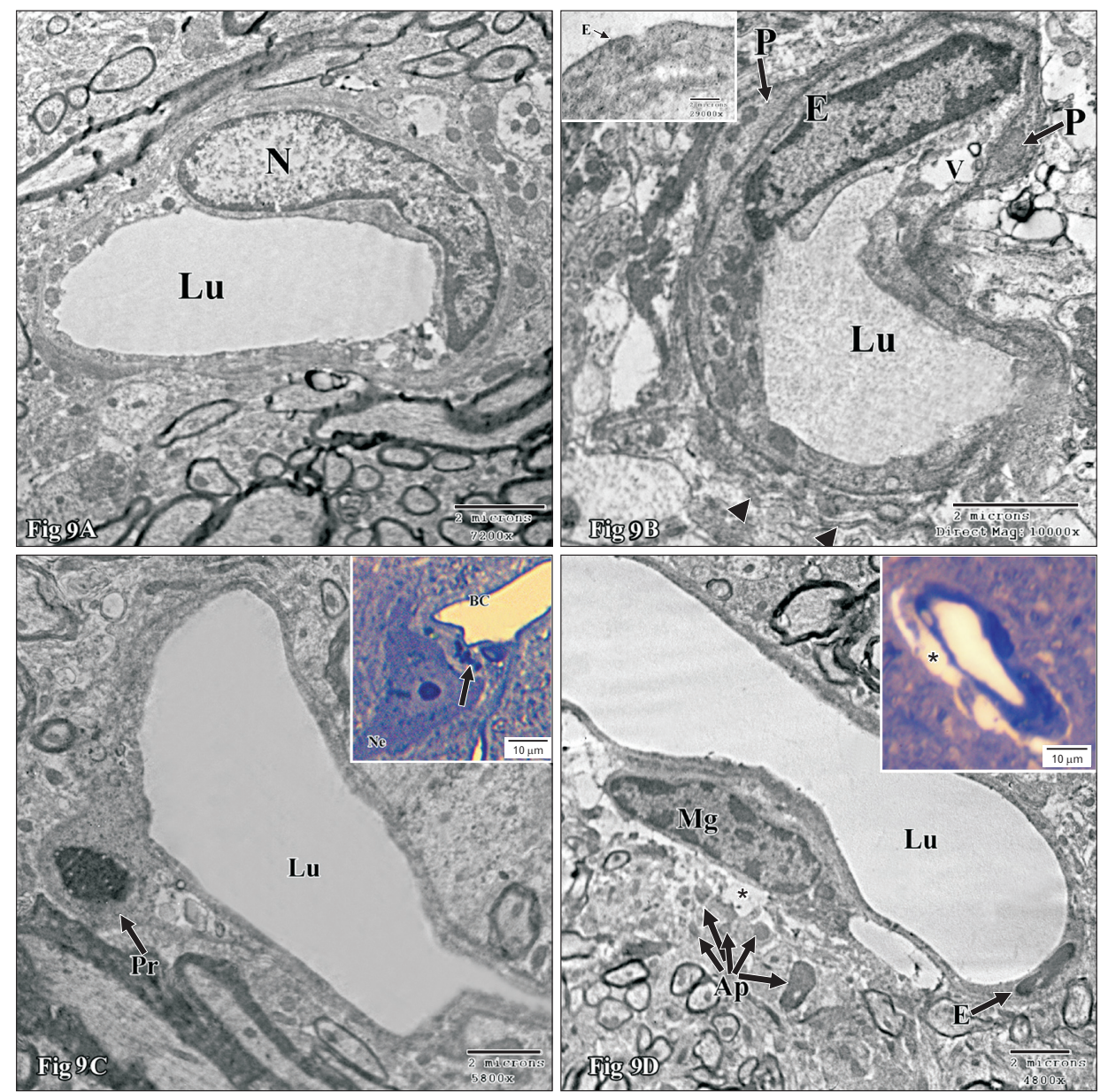

Fig. 9. (A) TEM of SNc of nicotine group showing a blood capillary lined by an E with an abnormally large and irregular N. (B) TEM of SNc of nicotine group showing a blood capillary with an irregular Lu. The $\mathrm{N}$ of the lining $\mathrm{E}$ has marginated heterochromatin and the surrounding astrocytic processes has corrugated surface $(\boldsymbol{\Delta})$. Inset: TEM of SNc of nicotine group showing a part of a blood capillary lining E with indistinct junctional complex. (C) TEM of SNc of nicotine group showing a blood capillary with Lu lined by a P with an apoptotic N (Pr). Inset: Semithin section showing apoptotic bodies $(\uparrow)$ between a BC and a dark Ne. (D) TEM of SNc of nicotine group showing a blood capillary with Lu, lined by an attenuated $\mathrm{E}$ with dense nucleus. The presence of the close by $\mathrm{Mg}$ with a rod-shaped nucleus and numerous apoptotic dense bodies can be detected. The capillary is surrounded by dilated astrocytic processes $\left({ }^{*}\right)$. Inset: Semithin section showing dilated astrocytic processes $\left({ }^{*}\right)$ around a blood capillary. Ap, apoptotic dense bodies; BC, blood capillary; E, endothelial cell; Lu, lumen; Mg, microglia; N, nucleus; Ne, neuron; P, pericytic process; Pr, pericyte; SNc, substantia nigra compacta; V, vacuole. 
by a scanty cytoplasm were occasionally observed (Fig. 8C). Astrocytic end feet processes around the blood capillaries might become swollen (Fig. 9D). Oligodendrocytes might be enlarged with numerous organelles and/or numerous dense inclusions and rod elongated nuclei (Fig. 8D) and usually encountered as two identical adjacent cells. Reactive microglia characteristically became hypertrophied with relatively electron-dense cytoplasm containing numerous electron dense bodies and elongated nuclei (Fig. 8E).

The blood capillaries in the control group were lined by highly flattened endothelial cells with flattened nuclei and a few organelles (Fig. 5A). Endothelial cells were connected by tight junctions and enveloped by pericytes. In nicotine treated group, the blood capillaries might show irregular lumen, attenuated endothelial cells and/or apoptotic pericytes (Fig. 9A). The blood capillaries might reveal an abnormally large irregular (Fig. 9A), attenuated or apoptotic endothelial lining and irregular lumina (Fig. 9B, C). In addition, the vascular endothelium might exhibit indistinct junctional complex or fenestrae (Fig. 9B). The surrounding pericytic (Fig. 9C) and/ or perivascular cells might also reveal apoptosis (Fig. 9D).

\section{Discussion}

This work revealed that nicotine use (11 days) might produce injurious effects on both histological structure and function of the SNc. This would influence the striatum function as DA neurons located in the SNc primarily project to form the so-called nigrostriatal pathway, which predominantly regulates motor function. In support with our suggestion, we found that nicotine use produced no increase in DA level (the increment was insignificant) and a decrease in immune reactivity for $\mathrm{TH}$, the marker for dopamine neurons. The literature also revealed that greater lifetime use of cigarettes (measured in pack-years) was associated with smaller left nucleus accumbens area volume $(P=0.018)$ and larger left putamen volume $(P=0.025)$ [13]. The presence of numerous electron-dense neurons revealing presence of multiple Nissle bodies along with prominent nucleoli (formed mainly of pars granulosa) indicate neuronal excitation. It is declared that nicotine exerts an excitatory action in cell body of neurons at the level of nigral DA nerve cells [26]. So, we propose that nicotine use in control animals might induce stimulation followed by exhaustive degeneration.

Reduced dopaminergic neurons, that we observed in nicotine treated group, occurred most probably by apopto- sis. This is confirmed by immunohistochemistry using anti caspase- 3 antibodies and by ultrastructure. The apoptotic features included an increase in neuronal electron density, their shrinkage and blebs of the neurons outlining. In addition, some cells exhibited breakdown of the Golgi apparatus which is considered an early event during apoptosis [27] as the Golgi localized caspase-2 and caspase-3 mediate cleavage of several Golgi-associated proteins That early event coincided with release of cytochrome $\mathrm{c}$ from mitochondria. Significantly, Golgi fragmentation preceded major changes in the organization of both the actin cytoskeleton and microtubules [28].

Coinciding with our findings numerous investigations carried on neuronal interactions of nicotine have highlighted the adverse potentials of this agent on some brain regions. In rat temporal cortices nicotine induced neuronal apoptosis [2]. In the prefrontal cortex, nicotine administration decreased the size of perikria and number of dendritic spines [29]. In addition; loss of cell layers thickness and their cellular constituents was induced in the cerebellum by nicotine [30]. Hippocampal and striatal neurodegenerative changes have been recorded as well under chronic nicotine administration [3]. In auditory cortex, nicotine produced an injurious dose related effect on the neurons [31].

Neuronal cells are specifically vulnerable to damage by peroxidation owing to their high oxygen utilization, high content of oxidizable polyunsaturated fatty acids, and the existence of redox-active metals $(\mathrm{Cu}, \mathrm{Fe})$ [32]. Decreased TAC detected in this work implies inability to inactivate the ROS. Along with the increase in MDA, the lipid peroxidation marker, we can suggest that neuronal activation for long period can result in their damage driven by biochemical alterations. In support of this suggestion, nicotine is found to block anti-oxidant enzymes, thereby increasing peroxidation of lipids, and formation of oxygen free radicals. Free radicals in the tissues are able to trigger cytoplasmic membrane damage and DNA fragmentation. An association between excessive formation of oxygen free radicals and the development of diseases such as tumors, hyperglycemia, arteriosclerosis, stroke, and neurodegenerative disorders has been reported. According to Cobley et al. [32], the rat temporal cortices revealed reduced antioxidant enzymes activity, and increased lipid peroxidation and oxygen free radicals. Moreover, plasma TAC level decreased in bidi workers as per exposure to nicotine [33], and the ROS levels increased in brain regions concerned with reward after recurrent exposure to a variety 
of misused drugs, including nicotine [34]. In addition, it has been reported that nicotine $(2.5 \mathrm{mg} / \mathrm{kg})$, reduced the total serum antioxidant capacity level with growth in the rate of ROS from nicotine at pancreases strictures of mice [35].

The positive reaction for antibodies against $\alpha$-synuclein observed in this study in nicotine group could be correlated with the presence of cytoplasmic assembles (aggregates) detected by electron microscopy and the secretory material detected inside dilated RER. So, we propose that chronic nicotine use might induce cytoplasmic aggregations and formation of inclusion bodies. Cytoplasmic inclusions more or less similar to those found here are formed following oxidative insult [36]. In their study, they tested the possibility that exposure of cells to patho-physiologically reasonable fluxes of reactive oxygen may induce the formation of protein aggregates in HEK 293 cells transfected with wild-type or mutant human $\alpha$-synuclein [36]. In further support of our suggestion neurons of high-expresser human $\alpha$-synuclein mice (2-3 months of age) showed electron-dense deposits (0.1-0.25 $\mathrm{mm}$ in diameter) associated with the RER [37]. Moreover, Miraglia et al. [38], has found that $\alpha$-Synuclein associates with specific membranes in neurons such as that of synaptic vesicles and some cellular organelles, like the RER/Golgi and the mitochondria. Since nicotine induced a decrease in TAC level, the aggregations of $\alpha$-synuclein could be attributed to the cellular adversities resulting from oxidative stress.

Under optimal conditions, $\alpha$-Synuclein functions in the normal brain as a soluble monomer. These are thought to mediate its physiological function in presynaptic terminals [39]. However, the forms of $\alpha$-synuclein oligomers and protofibrils are considered highly toxic. As they accumulate at presynaptic endings, they affect many steps of neurotransmitter release.

The dendro-dendritic reactive synaptogenesis detected in nicotine group, most probably, function to compensate for and/or restore partial functionality to the affected region as suggested by [40]. Those investigators proposed that this type of dendritic reactive synaptogenesis is thought to occur so as to re-saturate the region which has become vacant postsynaptic site following neurodegeneration caused by deafferentation or axotomy so that a partial functionality is restored [41].

Dilated astrocytic end-feet which surround the blood capillaries could probably be secondary to disrupted bloodbrain barrier. In support of this suggestion, Huang and his coworkers [12] reported that the blood circulating brain mi- crovascular endothelial cells (BMECs, an indicator of vascular damage), increased in mice after nicotine treatment [42]. So, dysfunction of BMECs, which are a major component of the $\mathrm{BBB}$, could be caused by nicotine.

Ischemia that might arise secondary to reduced blood flow could also account for the dilated astrocytic processes. This suggestion coincides with Petito and Babiak [43], work who reported that the most punctual reaction to anoxia-ischemia is conspicuous swelling of astrocytes which happens at first in end-feet around vessels. Since nicotine might induce vasoconstriction and consequently a decrease in cerebral blood flow (CBF) [44]. Loss of pericytes could result either directly from oxidative stress or indirectly through reduced cerebrovascular flow by nicotine. It is evident that pericytes are particularly sensitive to damage during pathological insults such as ischemia. According to Hall et al. [45], rat brain ischemia would induce persistent pericytes contraction and subsequent pericytes death. Pericytes have significant functions in $\mathrm{BBB}$ formation and maintenance, monitoring capillary diameter and CBF adjustment [46].

In conclusion, chronic nicotine administration in a dose ( $1.5 \mathrm{mg} / \mathrm{kg}$ body weight) might induce a negative influence on SNc. The reduced TAC, neuronal apoptosis and vascular adverse effects most probably were secondary to an oxidative stress.

\section{ORCID}

Sanaa A M Elgayar:

https://orcid.org/0000-0001-7365-6949

Ola A Hussein: https://orcid.org/0000-0002-4266-9840

Heba A Mubarak: https://orcid.org/0000-0002-0687-2745

Amany M Ismaiel: https://orcid.org/0000-0002-9422-3381

Asmaa M.S. Gomaa:

https://orcid.org/0000-0003-0985-1891

\section{Author Contributions}

Conceptualization: SAME. Data acquisition: SAME, OAH, HAM, AMI, AMSG. Data analysis or interpretation: SAME, OAH, HAM, AMI, AMSG. Drafting of the manuscript: SAME. Critical revision of the manuscript: SAME. Approval of the final version of the manuscript: all authors. 


\section{Conflicts of Interest}

No potential conflict of interest relevant to this article was reported.

\section{Acknowledgements}

Assiut University, Faculty of Medicine Research Grant is the source of funding for this research.

\section{References}

1. Zhou FM, Liang Y, Dani JA. Endogenous nicotinic cholinergic activity regulates dopamine release in the striatum. Nat Neurosci 2001;4:1224-9.

2. Hritcu L, Ciobica A, Gorgan L. Nicotine-induced memory impairment by increasing brain oxidative stress. Cent Eur J Biol 2009;4:335-42.

3. Ijomone OM, Olaibi OK, Esomonu UG, Nwoha PU. Hippocampal and striatal histomorphology following chronic nicotine administration in female and male rats. Ann Neurosci 2015;22:316.

4. Tewari A, Hasan M, Sahai A, Sharma PK, Rani A, Agarwal AK. White core of cerebellum in nicotine treated rats- a histological study. J Anat Soc India 2010;59:150-3.

5. Lin SJ, Hong CY, Chang MS, Chiang BN, Chien S. Long-term nicotine exposure increases aortic endothelial cell death and enhances transendothelial macromolecular transport in rats. Arterioscler Thromb 1992;12:1305-12.

6. Hans FJ, Wei L, Bereczki D, Acuff V, Demaro J, Chen JL, Otsuka T, Patlak C, Fenstermacher J. Nicotine increases microvascular blood flow and flow velocity in three groups of brain areas. Am J Physiol 1993;265(6 Pt 2):H2142-50.

7. Chen JL, Wei L, Bereczki D, Hans FJ, Otsuka T, Acuff V, Ghersi-Egea JF, Patlak C, Fenstermacher JD. Nicotine raises the influx of permeable solutes across the rat blood-brain barrier with little or no capillary recruitment. J Cereb Blood Flow Metab 1995;15:687-98.

8. Wang L, McComb JG, Weiss MH, McDonough AA, Zlokovic BV. Nicotine downregulates alpha 2 isoform of $\mathrm{Na}, \mathrm{K}-\mathrm{ATP}$ ase at the blood-brain barrier and brain in rats. Biochem Biophys Res Commun 1994;199:1422-7.

9. Abbruscato TJ, Lopez SP, Mark KS, Hawkins BT, Davis TP. Nicotine and cotinine modulate cerebral microvascular permeability and protein expression of $\mathrm{ZO}-1$ through nicotinic acetylcholine receptors expressed on brain endothelial cells. J Pharm Sci 2002;91:2525-38.

10. Hawkins BT, Brown RC, Davis TP. Smoking and ischemic stroke: a role for nicotine? Trends Pharmacol Sci 2002;23:7882.

11. Hawkins BT, Abbruscato TJ, Egleton RD, Brown RC, Huber JD, Campos CR, Davis TP. Nicotine increases in vivo blood- brain barrier permeability and alters cerebral microvascular tight junction protein distribution. Brain Res 2004;1027:48-58.

12. Huang SH, Wang L, Chi F, Wu CH, Cao H, Zhang A, Jong A. Circulating brain microvascular endothelial cells (cBMECs) as potential biomarkers of the blood-brain barrier disorders caused by microbial and non-microbial factors. PLoS One 2013;8:e62164.

13. Das D, Cherbuin N, Anstey KJ, Sachdev PS, Easteal S. Lifetime cigarette smoking is associated with striatal volume measures. Addict Biol 2012;17:817-25.

14. Licheri V, Eckernäs D, Bergquist F, Ericson M, Adermark L. Nicotine-induced neuroplasticity in striatum is subregionspecific and reversed by motor training on the rotarod. Addict Biol 2020;25:e12757.

15. Gomez AM, Sun WL, Midde NM, Harrod SB, Zhu J. Effects of environmental enrichment on ERK1/2 phosphorylation in the rat prefrontal cortex following nicotine-induced sensitization or nicotine self-administration. Eur J Neurosci 2015;41:109-19.

16. Ryan RE, Ross SA, Drago J, Loiacono RE. Dose-related neuroprotective effects of chronic nicotine in 6-hydroxydopamine treated rats, and loss of neuroprotection in alpha4 nicotinic receptor subunit knockout mice. Br J Pharmacol 2001;132:16506.

17. Hill P, Haley NJ, Wynder EL. Cigarette smoking: carboxyhemoglobin, plasma nicotine, cotinine and thiocyanate vs selfreported smoking data and cardiovascular disease. J Chronic Dis 1983;36:439-49.

18. Massadeh AM, Gharaibeh AA, Omari KW. A single-step extraction method for the determination of nicotine and cotinine in Jordanian smokers' blood and urine samples by RP-HPLC and GC-MS. J Chromatogr Sci 2009;47:170-7.

19. Yang L, Beal MF. Determination of neurotransmitter levels in models of Parkinson's disease by HPLC-ECD. Methods Mol Biol 2011;793:401-15.

20. Rabus M, Demirbağ R, Sezen Y, Konukoğlu O, Yildiz A, Erel O, Zeybek R, Yakut C. Plasma and tissue oxidative stress index in patients with rheumatic and degenerative heart valve disease. Turk Kardiyol Dern Ars 2008;36:536-40.

21. Tukozkan N, Erdamar H, Seven I. Measurement of total malondialdehyde in plasma and tissues by high-performance liquid chromatography and thiobarbituric acid assay. Firat Tip Dergisi 2006;11:88-92.

22. Azar HA, Maleki SA. Comparison of the anesthesia with thiopental sodium alone and their combination with Citrus aurantium L. (Rutaseae) essential oil in male rat. Bull Env Pharmacol Life Sci 2014;3:37-44.

23. Hayat MA. Principles and techniques of electron microscopy: biological applications. 4th ed. New York: Cambridge University Press; 2000.

24. Garth A. Analysing data using SPSS. Sheffield: Sheffield Hallam University; 2008.

25. Porter AG, Jänicke RU. Emerging roles of caspase-3 in apoptosis. Cell Death Differ 1999;6:99-104.

26. Lichtensteiger W, Hefti F, Felix D, Huwyler T, Melamed E, 
Schlumpf M. Stimulation of nigrostriatal dopamine neurones by nicotine. Neuropharmacology 1982;21:963-8.

27. Mukherjee S, Chiu R, Leung SM, Shields D. Fragmentation of the Golgi apparatus: an early apoptotic event independent of the cytoskeleton. Traffic 2007;8:369-78.

28. Mukherjee S, Shields D. Nuclear import is required for the pro-apoptotic function of the Golgi protein p115. J Biol Chem 2009;284:1709-17.

29. Jalili C, Salahshoor MR, Khademi F, Jalili P, Roshankhah SH. Morphometrical analysis of the effect of nicotine administration on brain's prefrontal region in male rat. Int J Morphol 2014;32:761-6.

30. Omotoso GO, Babalola FA. Histological changes in the cerebelli of adult wistar rats exposed to cigarette smoke. Niger J Physiol Sci 2014;29:43-6.

31. Elgayar SA, Hussein OA, Abdel-Hafez AM, Thabet HS. Nicotine impact on the structure of adult male guinea pig auditory cortex. Exp Toxicol Pathol 2016;68:167-79.

32. Cobley JN, Fiorello ML, Bailey DM. 13 reasons why the brain is susceptible to oxidative stress. Redox Biol 2018;15:490-503.

33. Sawant SD, Katkam R, Bhalshankar N. Occupational exposure of tobacco and its effect on total antioxidant capacity in bidi workers. Int J Biotechnol Biochem 2016;12:173-83.

34. Biala G, Pekala K, Boguszewska-Czubara A, Michalak A, KrukSlomka M, Grot K, Budzynska B. Behavioral and biochemical impact of chronic unpredictable mild stress on the acquisition of nicotine conditioned place preference in rats. Mol Neurobiol 2018;55:3270-89.

35. Salahshoor MR, Mirzaei F, Roshankhah S, Jalili P, Jalili C. Genistein improve nicotine toxicity on male mice pancreas. Anat Cell Biol 2019;52:183-90.

36. Paxinou E, Chen Q, Weisse M, Giasson BI, Norris EH, Rueter $\mathrm{SM}$, Trojanowski JQ, Lee VM, Ischiropoulos $\mathrm{H}$. Induction of alpha-synuclein aggregation by intracellular nitrative insult. J
Neurosci 2001;21:8053-61.

37. Masliah E, Rockenstein E, Veinbergs I, Mallory M, Hashimoto M, Takeda A, Sagara Y, Sisk A, Mucke L. Dopaminergic loss and inclusion body formation in alpha-synuclein mice: implications for neurodegenerative disorders. Science 2000;287:1265-9.

38. Miraglia F, Ricci A, Rota L, Colla E. Subcellular localization of alpha-synuclein aggregates and their interaction with membranes. Neural Regen Res 2018;13:1136-44.

39. Burré J. The synaptic function of $\alpha$-synuclein. J Parkinsons Dis 2015;5:699-713.

40. Eggers ED, McCall MA, Lukasiewicz PD. Presynaptic inhibition differentially shapes transmission in distinct circuits in the mouse retina. J Physiol 2007;582(Pt 2):569-82.

41. Hamori J. Morphological plasticity of postsynaptic neurones in reactive synaptogenesis. J Exp Biol 1990;153:251-60.

42. Liu L, Yu J, Li L, Zhang B, Liu L, Wu CH, Jong A, Mao DA, Huang SH. Alpha7 nicotinic acetylcholine receptor is required for amyloid pathology in brain endothelial cells induced by Glycoprotein 120, methamphetamine and nicotine. Sci Rep 2017;7:40467.

43. Petito CK, Babiak T. Early proliferative changes in astrocytes in postischemic noninfarcted rat brain. Ann Neurol 1982;11:5108.

44. Domino EF, Minoshima S, Guthrie S, Ohl L, Ni L, Koeppe RA, Zubieta JK. Nicotine effects on regional cerebral blood flow in awake, resting tobacco smokers. Synapse 2000;38:313-21.

45. Hall CN, Reynell C, Gesslein B, Hamilton NB, Mishra A, Sutherland BA, O'Farrell FM, Buchan AM, Lauritzen M, Attwell D. Capillary pericytes regulate cerebral blood flow in health and disease. Nature 2014;508:55-60.

46. Hamilton NB, Attwell D, Hall CN. Pericyte-mediated regulation of capillary diameter: a component of neurovascular coupling in health and disease. Front Neuroenergetics 2010;2:5. 